\title{
Research on the Knowledge Visualization teaching based on the Double-Whiteboard
}

\author{
Zhaoli Zhang, a , Zhifei $\mathrm{Li}^{2, \mathrm{~b}}$, Hai Liu ${ }^{1, \mathrm{c}}$ and Jiangbo Shü, d \\ ${ }^{1}$ National Engineering Research Center for E-Learning, Central China Normal University \\ 430079, Wuhan, China \\ ${ }^{2}$ Collaborative \& Innovative Center for Educational Technology, Central China Normal University \\ 430079, Wuhan, China \\ azl.zhang@mail.ccnu.edu.cn, ${ }^{b} 18766362 @ q q . c o m,{ }^{c h}$ hailiu0204@gmail.com, \\ dshujiangbo@mail.ccnu.edu.cn
}

Keywords: Knowledge visualization, Double-Whiteboard, Teaching research.

Abstract. Knowledge visualization plays an increasingly important role in the field of educational communication. However, most researches follow with interest a specific subject knowledge. Accordingly, we present the knowledge visualization by using different visualization tools based on the knowledge classification. And we present information in text and visualization by Double-Whiteboard. Then we selected two Physics classes as subjects in a middle school to reveal the impact of KV compared to traditional teaching. The result shows that this teaching method can effectively improve the teaching effects.

\section{Introduction}

Knowledge visualization (KV) based on computer has become extremely popular in recent years. And it has been widely used in the class of science education [1], marine zoology [2], mathematical concepts [3] and so on, which gets a lot of positive results. For students, they can obtain more attention, enjoyment and engagement in the class [4]. And for teachers, this kind of teaching method make the class become extra interactive and efficient [5]. Thus, it is a great way to reform the teaching mode.

However, there are still many issues with regard to KV. For instance, most researches follow with interest a specific subject knowledge such as Physics or mathematics. The application of alternative subject is really poor. Accordingly, it is necessary to promote it to other subjects. We are able to establish a method which can support most subjects to achieve visualization teaching.

In this paper, we present that visualizing the knowledge by using different visualization tools based on the knowledge classification. And showing on the Double-Whiteboard (DW) by combining text with $\mathrm{KV}$, which has many positive teaching advantages [6]. We will give some examples in the paper.

The outline of the paper is organized as follows. In the next section, we introduce the concept of $\mathrm{KV}$ and how to classify the knowledge which can be visualized. The examples showing on the DW combined text with KV are presented in Section 3. And KV teaching effect shows in Section 4, followed by a brief conclusion section.

\section{Knowledge Visualization and Classification}

Knowledge Visualization. Knowledge is growing rapidly with the development of human society. How to improve our learning efficiency will become very meaningful? The $\mathrm{KV}$, as a new direction of teaching research, is developed on the basis of scientific computing visualization, data visualization and information visualization, which is applicable to improve students' learning outcome. Fig. 1 shows the development of visualization.

Domestic and foreign researchers have done a lot of in-depth research regarding to KV. In July 2004, Martin J. Eppler and Remo A. Burkhard presented the concept of KV. They believed that the $\mathrm{KV}$ is to use of visual methods to communicate the formation of ideas, experiences, methods and techniques between two or more people. In China, Chen $\mathrm{H}$, Zhao $\mathrm{G}$ and $\mathrm{Xu} \mathrm{N}$ advanced the 
reformative concept of knowledge visualization. They considered that $\mathrm{KV}$ is a discipline which studies how visual representation can improve the transfer and innovation of complex knowledge between two or more people [7].

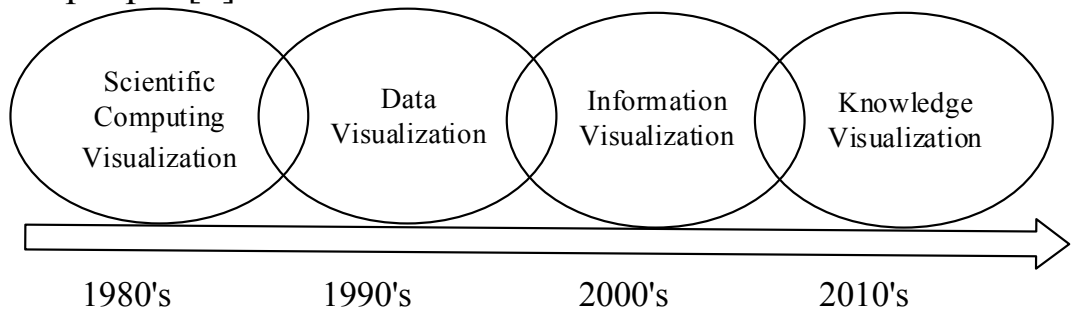

Fig. 1. Development of visualization.

We can summarize the two crucial functions of KV from these researches in Fig. 2. On the one hand, KV promotes knowledge's transfer. Knowledge is transformed into visual form by means of $\mathrm{KV}$, which reduce the cognitive difficulty of knowledge and help individuals improve their cognitive ability or understanding of the link between knowledge. On the other hand, KV is constructive for thinking inspiration. From the visualization technology to the visualization teaching, is not the straightforward technical application or the expansion of visualization domain. More importantly, it is the transformation of teaching thinking and the way to solve the problem, which can be widely used to solve the problem of knowledge creation and information overload.

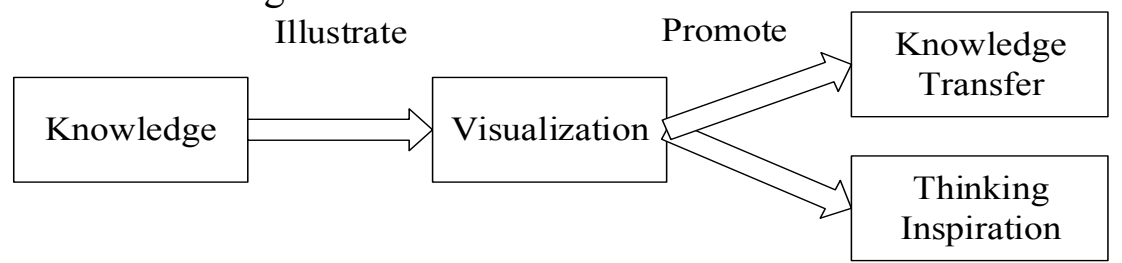

Fig. 2. Two crucial functions of KV.

Knowledge Classification. A sea of researches on KV show that different knowledge types need to use different visualization tools $[6,8]$. KV technology is developing rapidly. From the simple lines, charts and maps finished by hand to the animation, simulation and interactive visualization rely on computers, the degree of visualization is constantly improving and the knowledge that can be visualized is also constantly enriched. Accordingly, none of the visualization tools can be applied to all types of knowledge. Only by selecting the appropriate tools according to the characteristics of knowledge type, we can achieve the outstanding learning results.

The classification of knowledge can be different according to the different standard. In the light of subject, knowledge can be divided into language, mathematics, physics, chemistry and so on. According to the way in which knowledge is acquired, knowledge can be divided into direct knowledge and indirect knowledge. And according to the knowledge's consciousness levels, it can be separated into perceptual knowledge and rational knowledge.

Table 1 Three types of knowledge

\begin{tabular}{cccc}
\hline Elements & $\begin{array}{c}\text { Conceptual Knowledge } \\
\text { ( what) }\end{array}$ & $\begin{array}{c}\text { Procedural Knowledge } \\
\text { (how) }\end{array}$ & $\begin{array}{c}\text { Application Knowledge } \\
\text { (do) }\end{array}$ \\
\hline \multirow{3}{*}{ Characteristics } & The conceptual & The procedural knowledge & The application \\
& knowledge about & about & knowledge about \\
"what is fact" & "how to do" & "doing something" \\
Example & Beijing is the capital & Photosynthetic process of & Electromagnetic \\
& of China & green plants & induction \\
Tools & CmapTool, & Visio, Metafora, & Flash, 3DMAX, \\
& MindManager & Insight Maker & Virtools \\
Instantiation & Concept map, & Mind mapping, & Animation, Interactive \\
& Cognitive map & Thinking map & Visualization \\
\hline
\end{tabular}

On the basis of the above knowledge classification and the visual communication process of $\mathrm{KV}$, we believe that visual knowledge can be divided into Conceptual Knowledge (what), Procedural Knowledge (how) and Application Knowledge (do). Each knowledge type explained in Table 1. According to the characteristics of classification knowledge, we can use different visualization tools. These tools include concept map, cognitive map, mind mapping, thinking map and so on. Of course, 
with the development of technology, there have been some animation, simulation and interactive visualization of teaching tools, and achieved positive learning results $[4,9,10]$.

\section{Knowledge Visualization and Double-Whiteboard}

Many studies have shown that the combination of text and visualization of teaching methods can produce better learning results. The DW can present information in text and visualization which enhance the understanding of knowledge and memory through the "dual coding" processing [11]. The teaching schematic diagram of DW shown in Fig. 3.

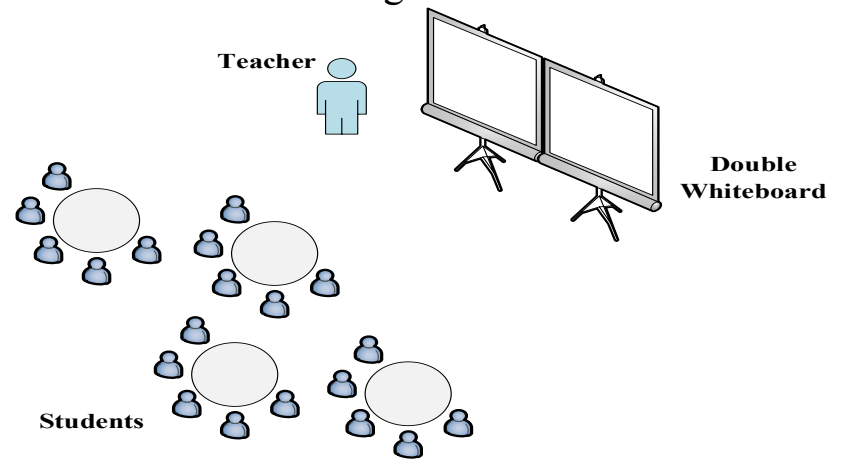

Fig. 3. Schematic diagram of DW.

Conceptual Knowledge Visualization. The concept map, presented by Dr. J.D. Novak of Cornell University in the United States, is based on the meaningful learning theory of David P. Ausubel. By using nodes, connections and other means to express the relationship and organization between concepts [12], which can be widely used for the learning and organization of Conceptual Knowledge.

It belongs to the Conceptual Knowledge about Chinese administration frame. Teachers can freehand sketch or use visualization tools to make the concept map, and then display on the DW. It's teaching diagram shown in Fig. 4. We divided the administrative regions of China into four parts, namely provinces, municipalities, autonomous regions and special administrative regions (marked in blue). Beijing is a municipality and the capital of China (marked in red). In this way, Chinese administration frame displays intuitively and completely in front of students.

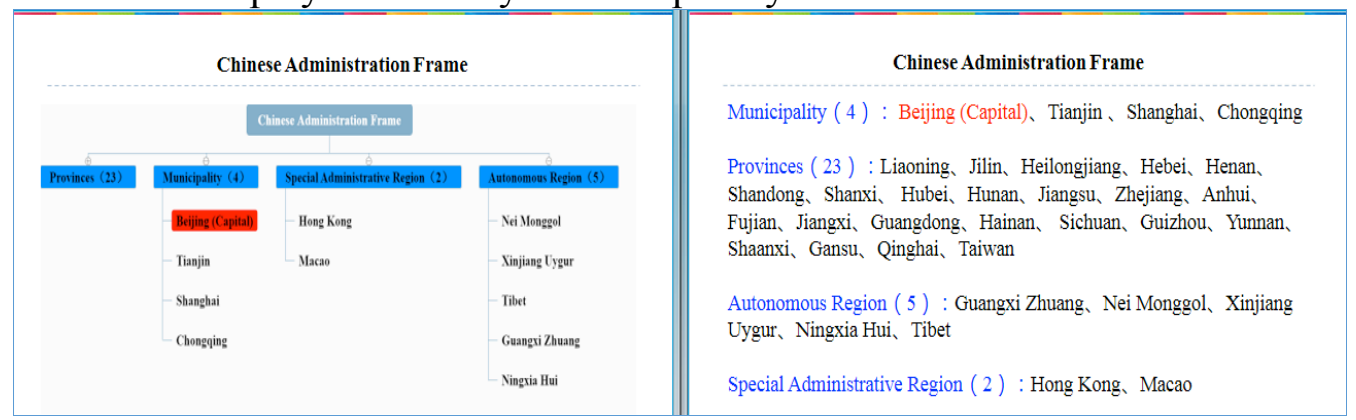

Fig. 4. DW teaching of Conceptual Knowledge.

Procedural Knowledge Visualization. Procedural Knowledge is to describe the dynamic change process of things, which reflects the basic facts, concepts, principles and rule of knowledge. This kind of knowledge is so complex that students can not directly perceive and learn. Teachers simply use language is difficult to accurately express. There are a lot of obstacles for students to understand, learn and master the relevant knowledge.

Modeling is also a form of representation of the KV. It can establish a simplified and clear model of what exists in the real world. The model can be manipulated and tested to achieve a real description of the relationship between the object [13]. We can use some basic visualization elements (objects, processes, variables, connections, etc.) to abstract Procedural Knowledge. And finally realize the visualization of such knowledge.

It's a good attempt to visualize the process of photosynthesis. Visio 2016 was used to visualize the process and shown in Fig. 5. After the visualization, we can clearly know that photosynthesis is divided into Light Reaction and Calvin Cycle. And each stage of the conditions, material conversion, energy conversion, etc. can be displayed to the students through the right screen. 


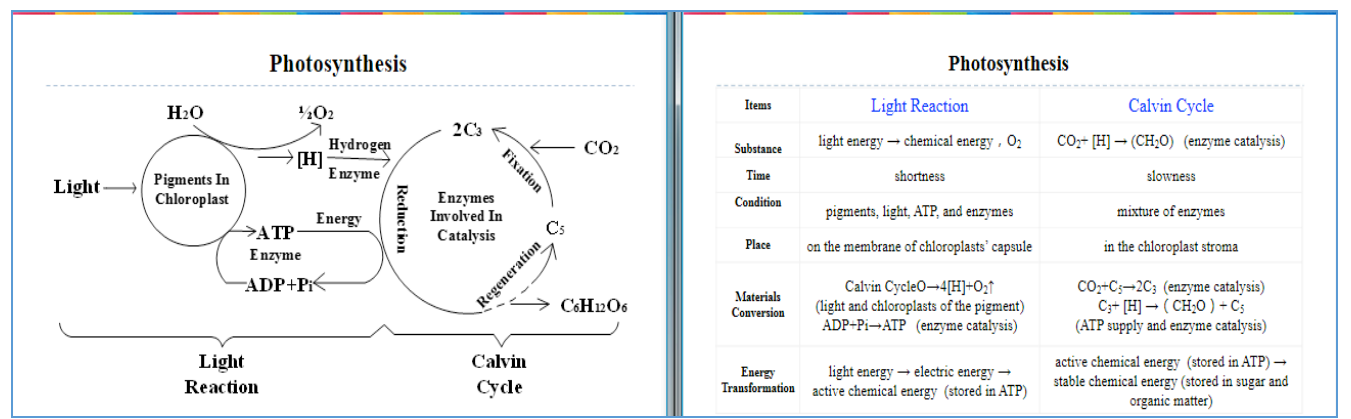

Fig. 5. DW teaching of Procedural Knowledge.

Application Knowledge. Application Knowledge is a process of inquiry, which emphasizes the ability of students to self-solve practical problems under the guidance of teachers. It can help students have an impressing understanding of knowledge and promote the students to deepen the knowledge. Interactive visualization provides methods to meet the students' self-inquiry learning requirements. And it has the characteristics of autonomy, process and practice. The ability to replace traditional teaching content with interactive visualization can help students achieve deeper and clearer understanding. And in the aspect of learning emotion, interactive visualization can improve the students' interest, motive and their enthusiasm in the teaching process.

Interactive whiteboard is a new type of teaching tools and means that increases man-machine interaction function on the basis of traditional blackboard and screen. An electromagnetic induction simulation experiment based on interactive whiteboard displays on the DW in Fig. 6. We can control the magnetic field direction, conductor cutting direction and conductor moving speed in this simulation experiment. And the modified changes can be dynamically displayed on the ammeter. Accordingly, students can operate the experiment in the left screen and read the experimental method and calculation formula in the right screen. Students will have a better learning effect about electromagnetic induction through this interactive visual learning method.

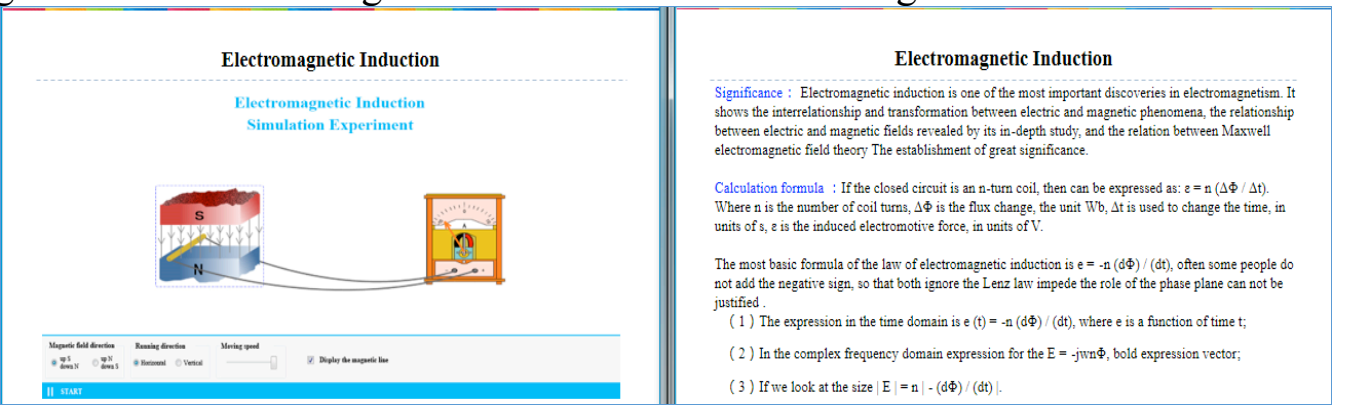

Fig. 6. DW teaching of Application Knowledge.

\section{Teaching Effectiveness Analysis of KV}

In order to reveal the impact of KV compared to traditional teaching. We selected two Physics classes as subjects in a middle school in Wuhan. One is the experimental class(KV) and the other is the control class(Traditional). And the experimentally independent variables were strictly controlled and balanced in the course of the study. After a month of teaching by two different methods. We had a test for both classes and the results are shown in Table 2 .

Table 2 Statistics of Physics

\begin{tabular}{|c|c|c|c|c|c|c|c|c|}
\hline & \multirow{3}{*}{$\mathrm{N}$} & \multirow{3}{*}{ M } & \multirow{3}{*}{ SD } & \multicolumn{5}{|c|}{ t-test for Equality of Means } \\
\hline & & & & \multirow{2}{*}{$\mathrm{t}$} & \multirow{2}{*}{ df } & \multirow{2}{*}{ Sig. (2-tailed) } & \multicolumn{2}{|c|}{$\begin{array}{c}95 \% \text { Confidence Interval } \\
\text { of the Difference }\end{array}$} \\
\hline & & & & & & & Lower & Upper \\
\hline KV & 48 & 52.15 & 14.25 & 2.199 & 90.0 & 0.030 & 0.673 & 13.255 \\
\hline Traditional & 44 & 45.18 & 16.12 & 2.187 & 86.2 & 0.031 & 0.635 & 13.293 \\
\hline
\end{tabular}

According to the data we can know that the significant probability of sig. (2-tailed) $p=0.030<$ 0.05. This result indicates that the KV teaching method in these two classes has significant difference, and the Mean of Physics score of students in KV is significantly higher than that of Traditional class. 
This experiment shows that KV can effectively promote the development of students, and this teaching method can effectively improve the teaching effects.

\section{Summary}

At present, the research of KV focuses on the specific knowledge content, which is not conducive to the development of visual teaching. This paper puts forward the idea of classifying the visual knowledge and adopting different visualization tools for different types. And we present information in text and visualization by DW. It can effectively improve the teaching effects through the result on Physics classes in a middle school.

With the development of information technology and computer technology, the way of KV will innovate constantly. For example, the education combined with Virtual Reality help us join into the "real classroom". The future will be the deep integration of technology and education.

Acknowledgment. The authors thank the editor and anonymous reviewers for their valuable suggestions. This research was partially funded by self-determined research funds of CCNU from the colleges' basic research and operation of MOE (CCNU16JYKX27), the National Natural Science Foundation of China under Grant (No. 61505064), the Project of the Program for National Key Technology Research and Development Program (2013BAH72B01, 2013BAH18F02, 2015BAH33F02), the Project of the Program for National Key Technology Research and Development Program (2014BAH22F01, 2015BAK07B03).

\section{References}

[1] M. Barak, T. Ashkar, and Y. J. Dori, "Learning science via animated movies: Its effect on students' thinking and motivation," Computers \& Education, vol. 56, pp. 839-846, 2011.

[2] B. Imhof, K. Scheiter, and P. Gerjets, "Learning about locomotion patterns from visualizations: Effects of presentation format and realism," Computers \& Education, vol. 57, pp. 1961-1970, 2011.

[3] H.-N. Liang and K. Sedig, "Can interactive visualization tools engage and support pre-university students in exploring non-trivial mathematical concepts?," Computers \& Education, vol. 54, pp. 972-991, 2010.

[4] S. Türkay, "The effects of whiteboard animations on retention and subjective experiences when learning advanced physics topics," Computers \& Education, vol. 98, pp. 102-114, 2016.

[5] J. Zhang, D. Zhong, and J. Zhang, "Knowledge Visualization: An Effective Way of Improving Learning," in Education Technology and Computer Science, 2010 Second International Workshop on, 2010, pp. 598-601.

[6] T. Kühl, K. Scheiter, P. Gerjets, and S. Gemballa, "Can differences in learning strategies explain the benefits of learning from static and dynamic visualizations?" Computers \& Education, pp. 176-187, 2011.

[7] H. Chen, G. Zhao, and N. Xu, "The analysis of research hotspots and fronts of knowledge visualization based on citespace II," in International Conference on Hybrid Learning, 2012, pp. 57-68.

[8] M. Patwardhan and S. Murthy, "When does higher degree of interaction lead to higher learning in visualizations? Exploring the role of 'Interactivity Enriching Features'," Computers \& Education, vol. 82, pp. 292-305, 2015.

[9] R. Lindgren, M. Tscholl, S. Wang, and E. Johnson, "Enhancing learning and engagement through embodied interaction within a mixed reality simulation," Computers \& Education, vol. 95, pp. 174-187, 2016.

[10] L. Lin and R. K. Atkinson, "Using animations and visual cueing to support learning of scientific concepts and processes," Computers \& Education, vol. 56, pp. 650-658, 2011.

[11] A. Paivio, "Mental Representations: A dual coding approach," Oxford Psychology, 1990.

[12] J. D. Novak and D. B. Gowin, Learning how to learn: Cambridge University Press, 1984.

[13] J. P. Sturmberg, L. Churilov, and G. Mcdonnell, "Modelling," Handbook of Systems \& Complexity in Health, vol. 38, pp. 81-111, 2013. 